\title{
城市地表温度与 NDVI 空间相关性的尺度效应
}

\author{
江颖慧 ${ }^{1,2}$, 焦利民 ${ }^{1,2 *}$, 张博恩 ${ }^{1,2}$
}

(1. 武汉大学资源与环境科学学院, 武汉 430079; 2 . 武汉大学地理信息系统教育部重点实验室, 武汉 430079)

\begin{abstract}
摘 要: 城市地表温度与NDVI的空间相关性已被广泛研究, 但是其尺度效应常常被忽略, 给研究结果带来不确定 性。本文以郑州市为例, 基于四幅 Landsat8 影像, 经辐射传导法反演地表温度(LST), 运用半变异函数识别地表温 度的空间相关性分析尺度,并结合空间相关指数 Moran's $I$, 从多尺度、多季节、多邻接范围 3 个方面对地表温度与 植被覆盖的空间相关性的尺度效应进行了探讨,结果表明: 1 LST 和NDVI的单变量空间自相关和双变量空间相 关尺度均在 300 m左右;(2)300 m 相关尺度内,单变量空间自相关性存在显著尺度效应,相比之下双变量空间相关 性尺度效应较为微弱; 3LST 和 NDVI 的单变量空间自相关性和双变量空间相关性尺度效应均表现出明显的季节 差异; (4) 随着邻接范围增大,LST 和 NDVI的空间自相关性减弱, 尺度效应更明显。因此度量 LST 和 NDVI的空间 相关性要考虑时空尺度效应, 本文研究结果有助于进一步认识 LST 和 NDVI间空间相关性的尺度效应。
\end{abstract}

关 键 词: 地表温度;NDVI; 半变异函数;空间相关性;尺度效应;郑州市

\section{1 引言}

城市热岛(Urban Heat Island, UHI)是城市城区 气温高于周边农村的现象(Howard, 1833), 是城市 气候最明显的特征之一。随着城市化进程的不断 加快, 城市热岛效应问题日趋严重。植被覆盖度与 地表温度均为描述生态系统特征的重要参数, 两者 间的关系一直是城市热岛相关研究的热点之一 (Makoto et al, 2001; Weng et al, 2004)。张小飞等 (2006)认为, 由于人为活动和下垫面类型的影响, 在 城市地域两者间的关系变得更为复杂, 下垫面性质 的改变是城市热岛效应增强的重要原因之一。热 环境遥感研究表明, 区域植被覆盖状况通过影响热 动力、热辐射以及土壤水分等多种地表特征, 导致 地表温度出现分异 (田平等, 2006; 武鹏飞等, 2009)。植被覆盖通过吸收和转化太阳辐射能量, 可有效地降低地表温度, 两者之间存在显著的负相 关关系(张穗等, 2003), 但这种关系不是简单的线性
关系, 且受季节影响(Yuan et al, 2007)。上述研究表 明, 植被覆盖对城市热环境具有积极的影响, 能缓 解城市热环境问题,但这种效果的显著程度因时空 分布而异,可能存在复杂非线性关系和尺度效应。

空间自相关分析是测试空间某点的观测值是 否与其相邻点的值存在相关性的一种分析方法(陈 小勇等, 2000)。目前, 空间自相关理论与方法已被 广泛应用于生态学(Sokal et al, 1978)、土壤学(倪万 魁等, 2002)、区域经济(陈斐等, 2002)等多方面的研 究。研究发现,对同一区域的某种地理现象在不同 分辨率(不同尺度)下进行空间自相关分析时,往往 出现不一致性,即所谓的“尺度效应”。尺度问题一 直是整个 GIS 和 RS 学术界研究的重点及难点, 备 受相关学者的关注。目前关于不同尺度下空间自 相关性的研究较多, 但对其尺度效应缺乏深人讨 论。李月臣等(2006)认为对于一个大区域选择单一 分辨率的遥感影像进行某种变化检测显然存在不 足, 并运用地统计学方法对合理选取遥感影像的空

收稿日期: 2017-12-13; 修订日期: 2018-04-26。

基金项目: 国家自然科学基金项目(41571385) [Foundation: National Natural Science Foundation of China, No.41571385]。 作者简介: 江颖慧(1994-), 女, 江西上饶人,硕士研究生,主要从事城市热岛与地学建模, E-mail: jiangyinghui@whu.edu.cn。 通讯作者:焦利民(1977-), 男, 河南安阳人, 教授, 博士生导师, 主要从事土地利用覆盖变化、城市扩展、空间分析等方面的研 究,E-mail: 1mjiao027@163.com。

引用格式: 江颖慧, 焦利民, 张博恩. 2018. 城市地表温度与 NDVI空间相关性的尺度效应[J]. 地理科学进展, 37(10): 1362-1370. [Jiang Y H, Jiao L M, Zhang B E. 2018. Scale effect of the spatial correlation between urban land surface temperature and NDVI[J]. Progress in Geography, 37(10): 1362-1370.]. DOI: 10.18306/dlkxjz.2018.10.006 
间分辨率进行了初步分析, 结论表明遥感数据分辨 率的选择应与研究区的具体特征相对应。柏延臣 等(2004)就不同尺度下遥感像元信息的空间自相关 性进行了初探, 结果表明相邻像元间的空间自相关 性远远强于相隔较远的像元。

在城市热环境研究方面, 有许多地表温度 (Land Surface Temperature, LST) 和 NDVI 的空间自 相关性的应用。游绚(2011)以福州盆地为研究区, 从多个角度对城市热环境的空间格局及尺度效应 进行分析,结果表明研究区地表温度的空间自相关 性随影像尺度的改变而改变, 城市热环境的空间格 局存在明显的尺度效应。关于地表温度和植被覆 盖度之间双变量空间相关性的研究大多只针对某 一尺度进行, 而地表温度与植被覆盖度是典型的空 间连续分布变量, 两者之间的关系是否存在一定的 空间尺度依赖性(韩贵锋等, 2011)有待证实。在不 同区域尺度上的多项研究均表明NDVI对地表温度 具有显著的负向影响(谢苗苗等, 2011), 但未详细描 述这种负向影响的尺度效应。Weng 等(2004)对美 国印第安纳州的研究发现地表温度与植被覆盖度 之间皮尔逊相关系数随分辨率的变化而变化, 但是 未对这种变化的规律和特点加以具体的分析和 归纳。

目前学术界关于地表温度和植被覆盖度相关 性的研究已较为丰富, 已有研究也表明两者空间相 关性具有尺度效应, 但较少关注两者之间空间相关 性的尺度效应及其季节差异。李斌等(2017)以郑泫 都市区为例对比分析 NDVI、NDMI 与地表温度间 的关系, 结果表明 NDVI-LST 和 NDMI-LST 空间负 相关性均比较明显, 且这种负相关性随尺度增加而 减小。但该研究主要是区别 NDVI、NDMI 与地表 温度关系的差异, 对其尺度效应的系统性分析还值 得进一步深人。地表温度既有随机性又有结构性, 在空间分布上具有某种程度的自相关性和连续性, 经典统计学方法以取样的独立性和随机性作为基 本假设, 采用经典统计学方法研究地表温度存在局 限性。地统计学方法是以区域化变量理论为基础, 以半变异函数为主要工具, 研究在空间上既有随机 性又有结构性的自然现象, 因此可采用地统计学方 法揭示地表温度的空间变异规律(陈公德等, 2008)。

综上, 关于地表温度和植被覆盖度的空间自相 关性、双变量空间相关性的研究缺少系统的尺度效 应分析。本文以郑州市中心城区为例, 基于 4 个季 节时点的 Landsat8 遥感影像反演地表温度和植被
覆盖, 运用半变异函数识别地表温度的空间相关性 分析尺度, 采用多尺度空间相关性分析方法探究地 表温度与植被覆盖空间相关性的尺度效应。

\section{2 数据与方法}

\section{1 研究区概况}

郑州是河南省省会, 位于东经 $112^{\circ} 42^{\prime} \sim 114^{\circ} 14^{\prime}$ 、 北纬 $34^{\circ} 16^{\prime} \sim 34^{\circ} 58^{\prime}$, 地处中国中部地区, 河南省中 部偏北, 黄河下游, 是中部地区重要的中心城市, 国 家重要的综合交通枢纽。全市总面积 $7446 \mathrm{~km}^{2}$, 地 貌复杂, 主要有山地、丘陵、平原 3 种类型, 地势总体 上呈由西南向东北逐渐降低的趋势, 海拔范围为 75 1494 m, 属北温带大陆性季风气候, 四季分明。 年平均气温 $15.6{ }^{\circ} \mathrm{C}$, 多年平均年降水量 $640 \mathrm{~mm}$, 降 雨大部分集中在 7、8、9月。近年来,郑州市人口、建 成区面积快速增长, 城市下垫面中的不透水地面增 多, 绿地减少, 城市热岛效应加剧(池腾龙, 2016)。 选取郑州市为研究对象具有一定的典型性。此外, 郑州市水体覆盖面积较小, 可较大程度地避免水体 对地表温度的影响。

\section{2 数据来源与处理}

参照季节划分标准(春季 3-5 月、夏季 6-8 月、秋 季9-11月、冬季 12-2 月), 综合考虑数据可获取性和 影像质量等因素以及最小时间跨度原则, 从地理空 间数据云(http://www.gscloud.cn/)获取了郑州市 4 期 高清无云 Landsat8 影像, 成像时间分别为 2014 年 5 月 6 日、2013年 6 月 4 日、 2015 年 9 月 14 和 2014 年 12 月 16 日, 分别代表春夏秋冬 4 个季节。其中 5 月和 6 月刚好处于郑州市主要农作物绿熟生长和收割完 毕的 2 个标志性阶段, 因此虽然这 2 个时点相近但 其地表覆盖的差异性显著, 2 个时点的影像数据可 以分别代表郑州市的春、夏 2 个季节地表覆盖特 征。对 4 幅影像数据进行辐射校正和裁剪等预 处理。

\section{3 研究方法}

\subsection{1 地表温度反演}

地表温度是衡量地球环境变化的重要参数之 一,而遥感技术是现代重要的对地观测手段。目前 国内外基于Landsat影像反演地表温度常用的方法 主要有大气校正法(辐射传导法)、单窗算法、单通道 算法等。针对不同的研究对象采用合适的算法,有 助于更准确地反演地表温度。本文基于 Landsat8 影像, 采用辐射传导法反演地表温度。在热红外遥 
感的地气辐射传输中, 卫星传感器接收到的热红外 波段辐射能量(亮度值) $L_{\lambda}$ 由 3 个部分组成: 大气上行 辐射亮度(大气直接热辐射) $L$ 、大气下行辐射亮度 (大气向地面的热辐射) $L_{\downarrow}$ 、地面的真实辐射亮度经 过大气衰减后被卫星传感器接收的热辐射能量(游 绚等, 2009) $L_{\mathrm{T}}, L_{\lambda}$ 可以表示为:

$$
L_{\lambda}=\left(\varepsilon L_{\mathrm{T}}+(1-\varepsilon) L_{\downarrow}\right) \tau+L_{\uparrow}
$$

式中: $\varepsilon$ 和 $\tau$ 分别为地表辐射率和大气透射率。假设 地表和大气对热辐射具有朗伯体性质, 根据式(1)可 推导出与地表真实温度相同的黑体在热红外波段 的辐射亮度 $L_{\mathrm{T}}$ :

$$
L_{\mathrm{T}}=\left(L_{\lambda}-L_{\uparrow}-\tau(1-\varepsilon) L_{\downarrow}\right) /(\tau \varepsilon)
$$

准确反演地表温度的关键在于准确估计式(2) 中的各个参数值。 $L_{\lambda}$ 通过对 Landsat8 影像的热红外 数据中第 10 波段进行辐射定标得到, 根据式(3)把 像元 DN 值转换为相应的表观辐射亮度。

$$
L_{\lambda}=G_{\text {ain }} \times D N+O f f_{\text {set }}
$$

式中: $G_{\text {ain }}$ 和 $O f f_{\text {set }}$ 是影像中自带的波段定标系数。 大气透射率 $\tau$ 、大气向上辐射亮度 $L$ 、和大气向下辐 射亮辐射亮度 $L$ 、可通过 NASA 官网(http://atmcorr. gsfc.nasa.gov)中输人影像成影时间、中心经纬度以 及其他相应的参数查询得到。

关于地表辐射率 $\varepsilon$ 的估计,将地表分成水体、自 然表面和城镇区, 分别针对 3 种地表类型计算地表 比辐射率, 具体算法参见覃志豪等(2004)。

将 $L_{\lambda} 、 L_{\uparrow} 、 L \downarrow \tau$ 和 $\varepsilon$ 代人式(2)得到 $L_{\mathrm{T}}$, 根据普朗克 公式的反函数,推导出地表真实温度 $T$ 的计算公式:

$$
T=K_{2} / \ln \left(K_{1} / L_{\mathrm{T}}+1\right)
$$

式中: $K_{1}, K_{2}$ 为常数, 对于 Landsat8 影像, $K_{1}=774.89$ $\mathrm{W} /\left(\mathrm{m}^{2} \cdot \mu \mathrm{m} \cdot \mathrm{sr}\right), K_{2}=1321.08 \mathrm{~W} /\left(\mathrm{m}^{2} \cdot \mu \mathrm{m} \cdot \mathrm{sr}\right)$ 。

\subsection{2 植被指数提取}

NDVI是监测地区或全球植被和生态环境变化 的有效指标(杨春华等, 2013)。利用植物在红光波 段和近红外波段反射光谱的明显差异, 计算归一化 值得到植被指数 NDVI:

$$
N D V I=(N I R-R) /(N I R+R)
$$

式中: $R 、 N I R$ 分别表示红光波段 Band 3 和近红外波 段 Band 4 的地面相对反射率。 $N D V I$ 的值介于 -1 和 1 之间,一般认为 $N D V I$ 大于 0 表示有植被覆盖, 其 值增加表示植被的增加, 超过 0.5 表明植被生长状 态好, 覆盖密度大。

\subsection{3 半变异函数}

半变异函数也称为半方差函数, 是地统计学中
用来描述区域化变量空间连续变异性质的函数,通 过测算两点空间属性的变异程度与两点的间距的 关系来描述要素的分布特征(册亭等, 2014)。半方 差表示给定空间距离间隔实测值两两之间差的平 方和的一半,一般来说半方差值会随着点对间距离 的增大而增大, 这是因为距离相近的样品点的性质 较为相似(地理学第一定律)。半变异函数 $\gamma(h)$ 随着 $h$ 的增大而单调递增, 当空间滞后距离 $h$ 超过某一 个范围时变异函数 $\gamma(h)$ 不再增大, 而是在一个极限 值附近摆动, 这种现象称为 “跃迁现象” (马恭博, 2014)。此时 $h$ 即为识别出的最佳操作尺度, 距离超 出 $h$ 的样品点对不再表现出空间自相关, 而在 $h$ 范 围内的物体间表现出一定程度的空间相关性 (Meisel et al, 1998)。在满足二阶平稳或本征假设条 件下(Bachmaier et al, 2011), 半变异函数的计算公式 为(Mejia-dominguez et al, 2012):

$$
\gamma(h)=\left(\frac{1}{2} N(h)\right) \sum_{i=1}^{N(h)}\left[Z\left(x_{i}\right)-Z\left(x_{i}+h\right)\right]^{2}
$$

式中: $\gamma(h)$ 是距离为 $h$ 的所有样本点对变异值的平均 值; $Z(x)$ 为区域化随机变量(本文中指 LST 或 NDVI 值); $h$ 为 2 样本点空间分隔距离; $N(h)$ 为空间距离为 $h$ 的样本点的数量; $Z\left(x_{i}\right) 、 Z\left(x_{i}+h\right)(i=1,2, \cdots, N(h))$ 分别 为区域化变量 $Z(x)$ 在空间位置 $x_{i}$ 和 $x_{i}+h$ 上的数值。 通过上述公式可以计算出任意给定距离 $h$ 内样本的 半变异值,绘出基于 $h-\gamma(h)$ 的半变异方差图。本文 基于半变异函数绘制研究区LST 和 NDVI的半变异 方差图, 从而确定两者的空间相关性尺度。

\subsection{4 多尺度空间相关性分析}

空间自相关是指同一个变量在不同空间位置 上的相关性, 是空间单元属性值聚集程度的一种度 量, 具有全局和局部 2 种指标, 常用的全局指标有全 局莫兰指数(Moran's $I$ )、全局 $\mathrm{G}$ 系数和基尔里 $\mathrm{C}$ 系 数(Geary $C$ ) 等。本文主要从全局的角度出发, 使用 Moran's $I$ 指数对郑州市主城区的地表温度和植被 覆盖度的空间关系进行研究。其单变量 Moran's $I$ 指数计算公式为(王劲峰, 2006):

$$
I=\frac{n}{\sum_{i} \sum_{j} \boldsymbol{w}_{i j}} \cdot \frac{\sum_{i} \sum_{j} \boldsymbol{w}_{i j}\left(x_{i}-\bar{x}\right)\left(x_{j}-\bar{x}\right)}{\sum_{i}\left(x_{i}-\bar{x}\right)^{2}}
$$

式中: $I$ 为 Moran's $I$ 指数; $n$ 为研究对象空间单元个 数; $x_{i} 、 x_{j}$ 分别为相邻研究区域 $i$ 和 $j$ 的属性值; $\bar{x}$ 为样 本中所有属性的平均值, $\boldsymbol{w}_{i j}$ 为空间单元 $i$ 和 $j$ 之间的 空间连接矩阵。 
其中Moran's $I$ 的取值在 -1 和 1 之间, $I>0$ 表示该 变量呈空间正相关性,其值越大, 空间正相关性越 明显; $I<0$ 表示空间负相关性, 其值越小, 空间负相 关性越明显; $I=0$ 表示呈空间随机性分布。

为满足对双变量乃至多变量之间的空间相关 性分析的需求, Wartenberg(1985)结合单变量空间相 关性的原理对 Moran's $I$ 指数公式(7)进行了改进, 提 出了 Multivariate Moran' $I$ 指数, 其中双变量 Moran's $I$ 指数的公式如下:

$$
I_{x y}=\frac{n}{\sum_{i} \sum_{j} \boldsymbol{w}_{i j}} \cdot \frac{\sum_{i} \sum_{j} \boldsymbol{w}_{i j}\left(x_{i}-\bar{x}\right)\left(y_{j}-\bar{y}\right)}{\sqrt{\sum_{i}\left(x_{i}-\bar{x}\right)^{2}} \sqrt{\sum_{j}\left(y_{j}-\bar{y}\right)^{2}}}
$$

式中: $x_{i}$ 为相邻研究区域 $i$ 的 $x$ 属性值; $y_{j}$ 为相邻区域 $j$ 的 $y$ 属性值; $\bar{x}$ 和 $\bar{y}$ 分别为样本中所有 $x$ 和 $y$ 的属 性平均值; $I_{x y}$ 为双变量 Moran's $I$ 指数, $I_{x y}$ 的取值范围 和意义均同上; 其他指标意义同上。

对影像进行预处理后经辐射传导法反演得到 地表温度(LST)并提取 NDVI值, 基于半变异函数确 定 LST 和 NDVI 的空间相关性尺度后, 在合理的尺 度范围内分析两者空间相关性的尺度效应。分别 对原始分辨率 (30 m) 的 LST 和 NDVI进行重采样得 到各尺度(分辨率)下的 LST 和 NDVI, 并提取值到 对应尺度的网格点。本文将LST 和 NDVI影像裁剪 为包含郑州市主城区的大小为 1200 像元 $\times 1200$ 像 元的子影像, 采用平均像元聚合的方法进行尺度变 换。在影像尺度变换过程中, 为保证影像大小一致 且像元不被分割与重组, 最终将尺度分别设置为 $30 \mathrm{~m} 、 60 \mathrm{~m} 、 90 \mathrm{~m} 、 120 \mathrm{~m} 、 150 \mathrm{~m} 、 180 \mathrm{~m} 、 300 \mathrm{~m}$, $600 \mathrm{~m} 、 900 \mathrm{~m} 、 1500 \mathrm{~m} 、 1800 \mathrm{~m}$ 和 $2250 \mathrm{~m}$ 等 12 组。 以网格点为基础, 运用空间相关性分析法, 建立 $\mathrm{K}$ 最
近邻空间权重矩阵,计算得到 4 个季度影像 12 个分 辨率下 LST 和 NDVI 各自的单变量 Moran's $I$ 值 $(I)$ 和两者间双变量 Moran's $I$ 值 $\left(I_{x y}\right)$ 。

\section{3 结果与分析}

\subsection{LST 和 NDVI相关性尺度的识别}

以郑州市 2014 年 5 月 6 日 (春季)的 Landsat8 影 像为例, 反演得到 LST 和NDVI 分布图(图 1)。由图 1 可以看出, 中心城区温度明显高于郊区温度, 说明 郑州市具有较为明显的热岛效应。NDVI的高值分 布在城郊区域,低值分布在城市中心区。已有研究 表明植被覆盖能有效缓解热岛效应, 图 1 在一定程 度上体现了NDVI对LST 的缓解作用。

相邻像元的属性具有较强的空间相关性, 但这 种空间自相关性将随像元间距的增加而逐渐减弱, 当像元间距增至某种程度时,这种空间相关性将变 得非常弱, 以致影像几乎丢失原有的信息而变成随 机影像, 不能再提供有意义的遥感信息(游绚, 2011)。因此为保证城市热环境研究严谨且有意义, 需运用地统计学工具识别出其最佳相关性尺度。 张小飞等(2006)以深圳市为例分析植被覆盖度与地 表温度的相关关系, 结果表明相关性在 $120 \mathrm{~m}$ 左右 达到最大, 即深圳市植被覆盖与地表温度最佳相关 尺度在 $120 \mathrm{~m}$ 左右。本节采用半变异函数识别 LST 和 NDVI有效相关性尺度。根据点对之间的空间滞 后距离 $h$ 将各季节 LST 和 NDVI半方差值绘制成半 变异方差图如图 2 所示。

半变异函数曲线主要包括块金值、基台值、偏 基台值和变程等 4 个参数,如图 $2 \mathrm{c}$ 所示: 块金值主
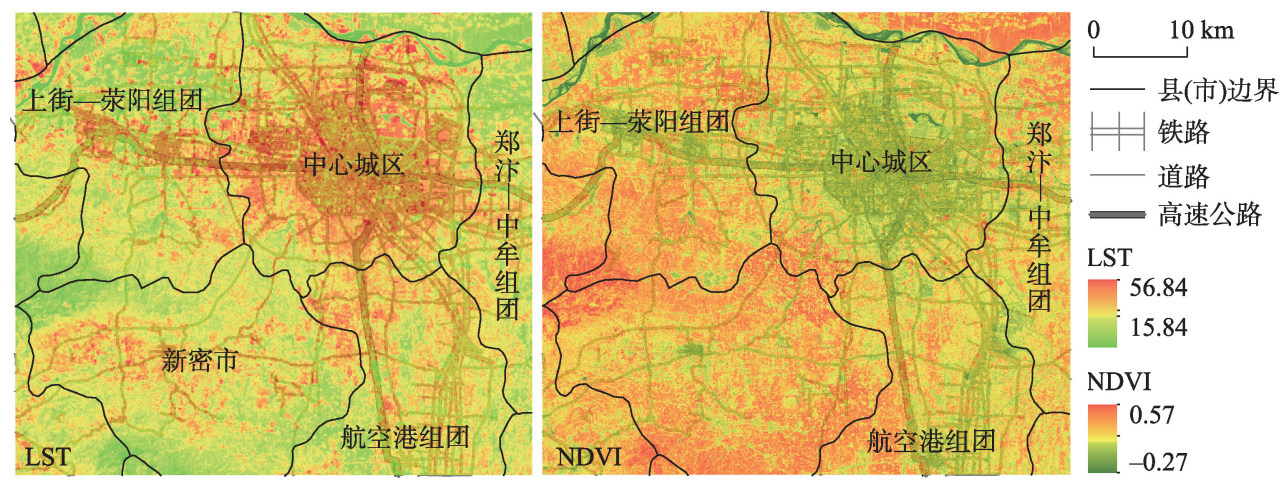

图 1 郑州市 LST 与 NDVI 分布图(春)

Fig.1 Spatial distribution of land surface temperature (LST) and Normalized Difference Vegetation Index (NDVI) in Zhengzhou City (Spring) 

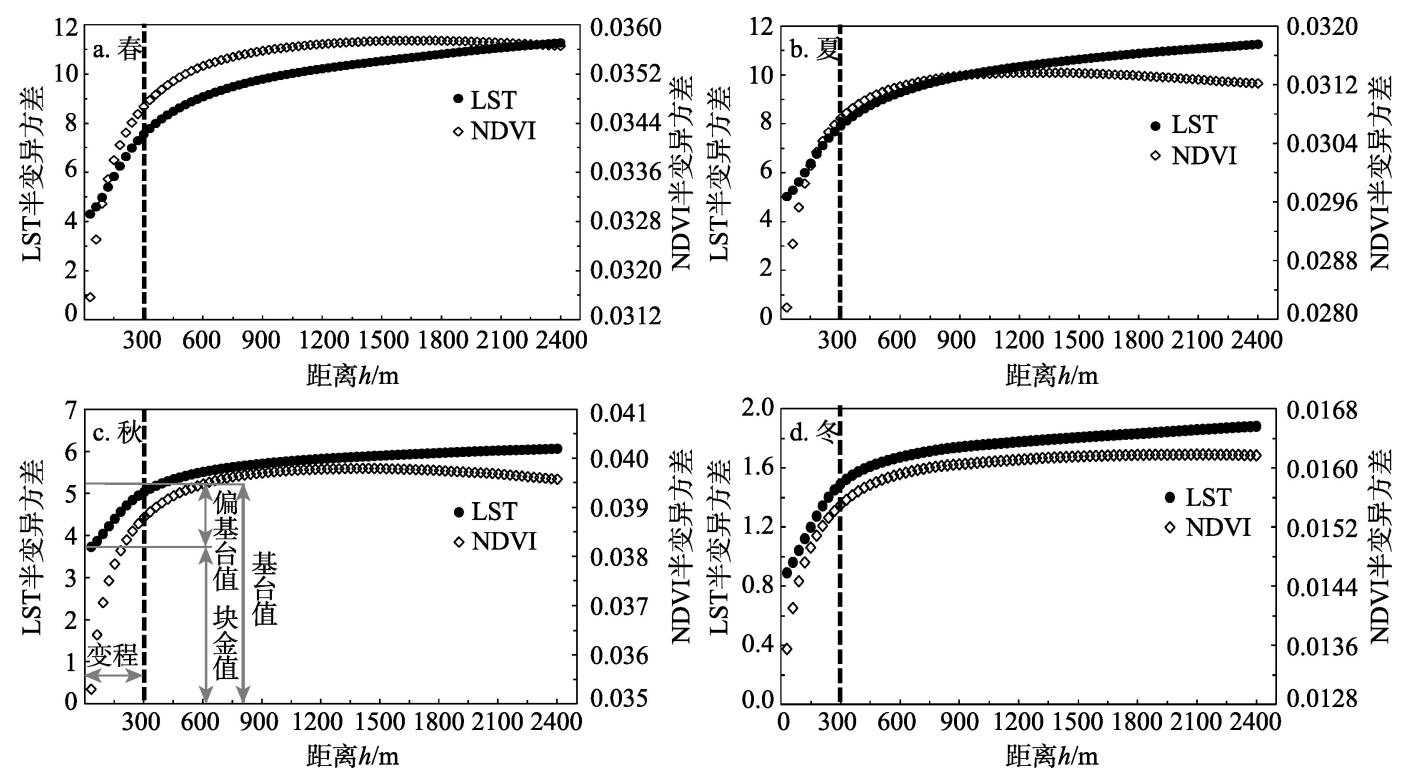

图 2 各季节LST 和NDVI的半变异方差图

Fig.2 Semivariance diagram of land surface temperature (LST) and NDVI of each season

要由测量误差和空间差异所致,使得非常相近的 2 个采样点之间的变异值不为 0 ; 基台值为半变异方 差随采样距离增加而增加最后达到的一个较为稳 定的值; 偏基台值为基台值与块金值的差值;变程 即为半变异方差由初始的块金值达到基台值时采 样点的间隔距离, 即本节需要识别出的空间相关性 尺度。图 2 中, 4 个季节的 LST 和 NDVI半变异方差 图均在整体上呈现先递增后平缓的变化趋势, 4 个 季节的 LST 和 NDVI的半变异函数曲线的变程均在 $300 \mathrm{~m}$ 左右。在 $300 \mathrm{~m}$ 尺度之前, 基台值随尺度增 加较快, $300 \mathrm{~m}$ 之后增幅明显减缓甚至不再增加, 说 明LST 和NDVI均逐渐由空间异质性趋向于空间同 质性。快速递增部分表明研究区 LST 和NDVI存在 较强的空间异质性, 该区间内 LST 和 NDVI均表现 出较强的空间自相关性。在 $300 \mathrm{~m}$ 范围内, LST 和 NDVI 具有较强的空间依赖性, 其空间自相关性尺 度效应较为明显, $300 \mathrm{~m}$ 以外两者不再表现出空间 自相关性。研究区半变异函数分析表明, 基于 Landsat8 数据的 LST 和 NDVI的空间自相关分析的 尺度应小于等于 $300 \mathrm{~m}$ 。

3.2 LST 和 NDVI 双变量空间相关性尺度效应的季 节差异

建立 $\mathrm{K}$ 最近邻空间权重矩阵 $(K=8)$, 即以 8 邻域 准则确定邻接范围, 分别计算 4 个季节 LST 与 NDVI 的单变量和双变量 Moran's $I$ 值。Z统计量绝对值均 大于 1.65 , 数据结果均有效且显著。研究区内各季
节 LST 和 NDVI 单变量空间自相关 Moran's $I$ 指数 $(I)$ 和双变量空间自相关 Moran's $I$ 指数 $\left(I_{x y}\right)$ 随尺度的 变化情况如图 3 所示。

图 3a为各季节 LST 单变量空间自相关性随着 分辨率增大的变化趋势图。总体而言, 各季节 LST 的单变量 Moran's $I$ 均为正值, 随着尺度的增大, 四 条曲线均呈下降的趋势, 表明研究区域内 4 个季节 LST 分布均呈现逐渐下降的空间正相关模式。此 外, $300 \mathrm{~m}$ 处曲线出现明显转折, $300 \mathrm{~m}$ 尺度后 LST 的空间自相关性呈现无规律的变化,进一步表明 $300 \mathrm{~m}$ 是较为合理的相关性尺度。在 $300 \mathrm{~m}$ 尺度范 围内, 原始分辨率 $(30 \mathrm{~m})$ 上 LST 的 Moran's $I$ 值高达 0.979 , 表现出相当强的空间自相关性, 随着尺度的 慢慢增大,各单元格之间进行了合并, LST 的空间 自相关性降低, 即研究区域单元格越大, LST 在区 域上的聚集程度逐渐降低,其相互间的依赖性逐渐 减小。300 m 尺度范围内, 秋季 LST 的 Moran's $I$ 值 水平始终最高, LST 的空间自相关性强度的季节差 异表现为: 秋 $>$ 春 $>$ 夏 $>$ 冬。LST 空间自相关性强度 的变化范围的季节差异为夏 $>$ 春 $>$ 冬 $>$ 秋, 即 LST 空 间自相关性强度的尺度效应由大到小依次为夏 $>$ 春 >冬>秋。

图 3b 中, 各季节 NDVI 单变量空间自相关性 随着分辨率增大的变化趋势与 LST 相似, 各季节 NDVI 的单变量 Moran's $I$ 均为正值且在 $300 \mathrm{~m}$ 尺度 范围内随尺度的增加而下降, 但整体变化幅度小于 

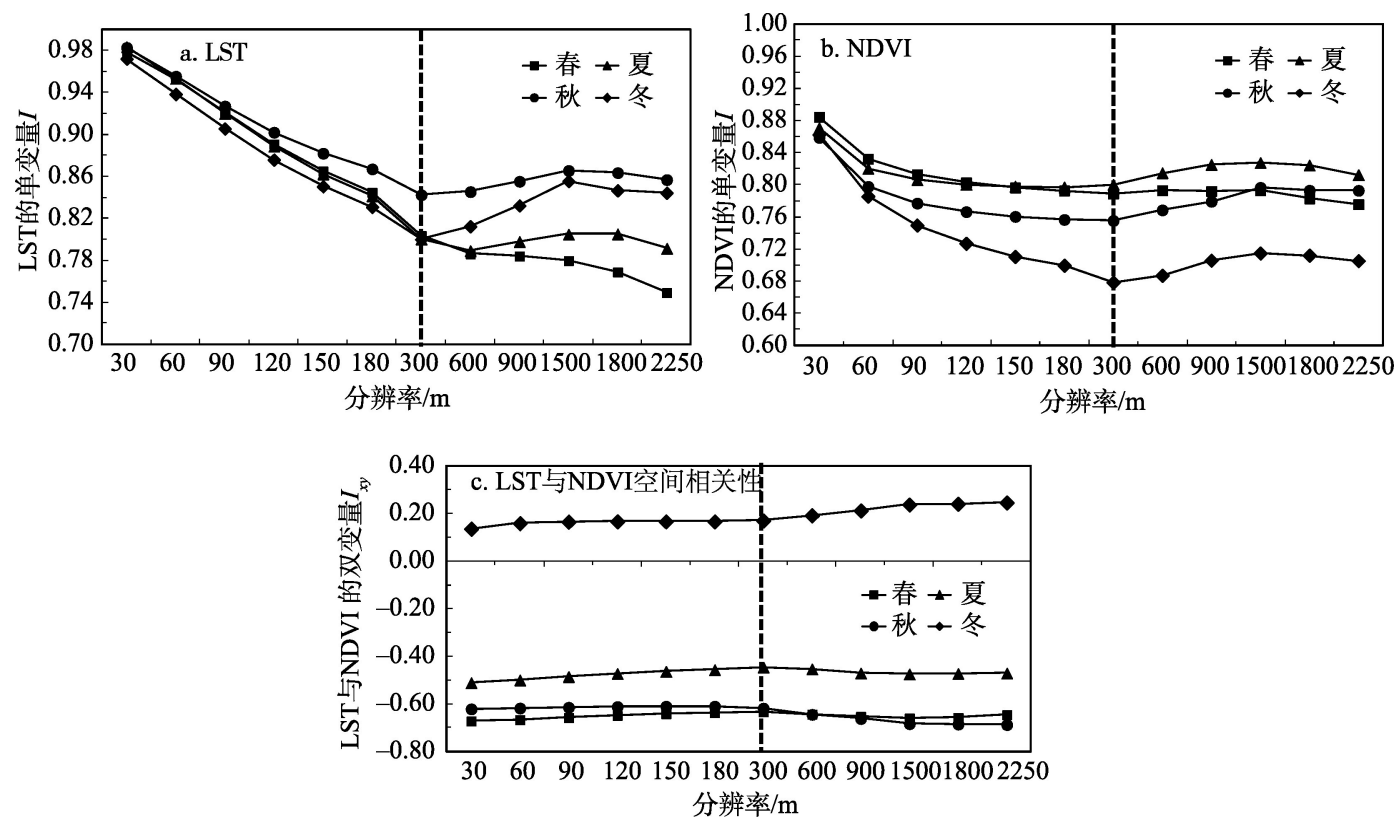

图 3 不同季节下LST(a)、NDVI(b)空间自相关性以及二者空间相关性(c)的尺度效应

Fig3. Scale effects of spatial autocorrelation of land surface temperature (LST) (a) and NDVI (b), and spatial correlation of the two (c) in different seasons

LST, 即各季节 NDVI 的空间自相关性尺度效应弱 于 LST。原始分辨率 NDVI 的 Moran's $I$ 值高达 0.884 , 同样表现出较强的空间自相关性。就 NDVI 空间自相关性强度的季节差异而言, 冬季 NDVI 的 空间自相关性强度始终处于最低水平, 其他 3 个季 节的强度较高且相差不大。就尺度效应的季节差 异而言, 夏季 NDVI 的 Moran's $I$ 值较其他 3 个季节 而言平均变化幅度最小, 尺度效应最小。

图 3c 中, 春夏秋 3 个季节的双变量 Moran's $I$ 值 均为负值, 表明春夏秋季的 LST 和 NDVI在空间上 呈现负相关性。冬季结果显示两者间的 Moran's I 值均为正值, 表明研究区内冬季的 LST 和 NDVI 呈 现空间正相关性。图 3c 还显示, 整体而言, 随着尺 度的增大各曲线均无较大起伏。夏季的双变量空 间相关性尺度效应最强, 但其变化幅度仅有 0.07 。 但是在 $300 \mathrm{~m}$ 尺度范围内, 4 个季节的双变量空间 相关性均存在轻微的下降趋势。由此可见, 双变量 空间相关性存在微弱的尺度效应, 且在 $300 \mathrm{~m}$ 尺度 范围内二者双变量空间相关性变化趋势与单变量 空间自相关性是一致的。

由上述分析可知, 双变量空间相关性尺度效应 较为微弱, LST 和 NDVI 的单变量空间自相关性具 有显著的尺度效应且 2 种空间相关强度均随尺度增 大而减小。此外, LST 和 NDVI 的单变量空间自相
关性尺度效应和双变量空间相关性尺度效应均存 在季节差异:夏季 LST 单变量空间自相关性尺度效 应最强,而 NDVI单变量空间相关性尺度效应最弱; 双变量空间相关性的尺度效应也在夏季达到最强。

\section{3 邻接范围对空间自相关性尺度效应的影响}

3.2 小节表明双变量空间相关性较为微弱, 因 此本节只探讨邻接范围对单变量空间自相关性尺 度效应的影响。以郑州市 2014 年 5 月 6 日的 Landsat8 影像为例, 在 3.2 节的基础上, 改变 $\mathrm{K}$ 最近邻空 间权重矩阵的 $K$ 值, 分别计算 $K=8(3 \times 3$ 网格 $) 、 K=24$ $(5 \times 5$ 网格 $)$ 和 $K=48(7 \times 7$ 网格 $)$ 时各个尺度下的 Moran's $I$ 值(下文分别简称为 8 邻域、 24 邻域和 48 邻域), 分析不同邻接范围下 LST 和 NDVI空间自相 关性的尺度效应。计算结果显示均通过 0.05 的显 著性水平检验, 且 $Z$ 统计量绝对值均大于 1.65 , 数据 结果有效。不同邻接范围下 LST 和 NDVI 单变量 Moran's $I$ 指数随尺度的变化情况如图 4 所示。

图 $4 a 、 4 b$ 分别为 3 种邻接范围下 LST 和 NDVI 单变量 Moran's $I$ 值随尺度变化的趋势图。 $300 \mathrm{~m}$ 尺 度范围内两者的 Moran's $I$ 值均为正值, 空间自相关 强度均高达 0.7 且每条曲线均随着分析尺度的增大 逐渐下降。LST 和 NDVI 的 8 邻域 Moran's $I$ 值均处 于最高的水平, 24 邻域次之, 48 邻域始终在最低水 平。对 Moran's $I$ 值的变化范围而言: 48 邻域最大, 

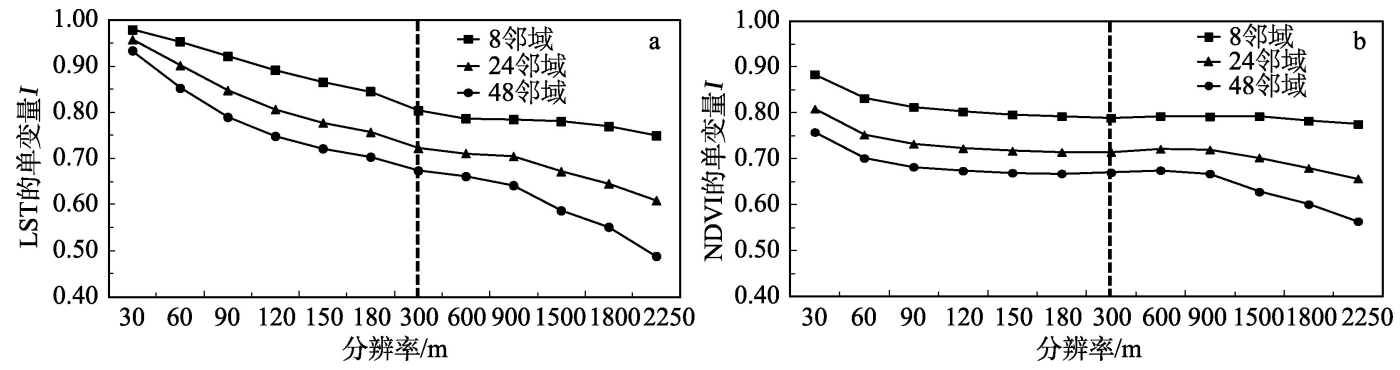

图 4 不同邻接范围下 LST(a)和NDVI(b)空间自相关性的尺度效应

Fig.4 Scale effects of spatial autocorrelation of land surface temperature (LST) (a) and NDVI (b) in different adjacent range

24 邻域次之, 8 邻域最小。表明在研究区范围内, 3 种邻域范围的 LST 和 NDVI 均存在正向的空间自相 关性且空间自相关性均随尺度的增大而变小。LST 和NDVI的空间自相关性的强度随邻接范围的增大 而变小,其空间自相关性的尺度效应随邻接范围的 增大而增大。

综上, 分析的邻接范围不同, 单变量空间自相关 性对于尺度变化的感知程度有所不同。在 $300 \mathrm{~m}$ 尺 度范围内, LST 和 NDVI的空间自相关性强度水平随 邻域范围增大而变小, 其自相关性的尺度效应随邻 域范围增大而增大。因此得出结论, 分析的邻接范 围越小, 空间自相关性的强度越大, 其尺度效应越 弱; 反之, 空间相关性强度越小, 其尺度效应越强。

\section{4 结论与展望}

本文以郑州市为例, 运用半方差图、空间自相 关指数和双变量空间相关指数, 从多尺度、多季节、 多邻接范围 3 个方面对地表温度与植被覆盖的空间 相关性的尺度效应进行了探讨, 得出如下结论：

(1) 对基于 Landsat8 影像生成的 LST 和 NDVI, 无论单变量空间自相关性还是双变量空间相关性, 其空间相关尺度相同, 均为 $300 \mathrm{~m}$ 。半变异方差图 和 LST 与 NDVI单变量空间自相关性尺度效应图均 证实了这一点。

(2) $300 \mathrm{~m}$ 相关尺度内, 单变量空间自相关性存 在显著尺度效应, 双变量空间相关性尺度效应较为 微弱, 且 2 种相关性相关强度均随尺度增大而减小。

(3) LST 和 NDVI 的单变量空间自相关性和双 变量空间相关性的尺度效应均存在季节差异。其 中, 夏季 LST 单变量空间自相关性尺度效应最强, 而 NDVI 单变量空间相关性尺度效应最弱, 双变量 空间相关性的尺度效应也在夏季达到最强。

(4) 邻接范围会对 LST 和 NDVI 的空间自相关
性的尺度效应产生影响。在 $300 \mathrm{~m}$ 有效尺度范围 内, 随着邻接范围增大, 空间自相关性减弱, 尺度效 应更明显。

本文系统分析了郑州市 LST 和 NDVI 的单变量 空间自相关性和双变量空间相关性的尺度效应以 及不同季节和邻接范围对这种尺度效应的影响, 有 助于更全面地认识 LST 和NDVI空间相关性度量的 尺度效应, 但也存在一些不足之处有待进一步完 善: 一是本文只选取了郑州市作为研究对象, 今后 可扩充其他地区的典型城市进行探讨以提高研究 结论的普遍性; 二是本文中地表温度数据基于 Landsat8 影像采用辐射传导法反演而来,与真实地 表温度可能存在差异。但目前地表温度反演方法 已较为成熟, 数据能够满足研究需求; 三是本文基 于数理统计的角度对 LST 和 NDVI空间相关性的尺 度效应进行定性分析, 揭示现象并总结规律, 对于 LST 和NDVI 在不同尺度上空间相关性的定量分析 以及两者间相互作用的空间机理还有待进一步 探究。

\section{参考文献(References)}

柏延臣, 王劲峰. 2004. 基于特征统计可分性的遥感数据专 题分类尺度效应分析 [J]. 遥感技术与应用, 19(6): 443449. [Bo Y C, Wang J F. 2004. Exploring the scale effect in thematic classification of remotely sensed data: The statistical separability- based method[J]. Remote Sensing Technology and Application, 19(6): 443-449.]

陈斐, 杜道生. 2002, 空间统计分析与 GIS 在区域经济分析中 的应用 [J]. 武汉大学学报: 信息科学版, 27(4): 391-396. [Chen F, Du D S. 2002. Application of the integration of spatial statistical analysis with GIS to the analysis of regional economy[J]. Geomatics and Information Science of Wuhan University, 27(4): 391-396.]

陈公德, 徐建华, 戴晓燕, 等. 2008. 运用遥感数据挖掘解析 城市地表温度的空间变异规律 [J]. 遥感技与应用, 23(4): 
405-409. [Chen G D, Xu J H, Dai X Y, et al. 2008. Applying Geo-data Mining to analysis spatial variance characters of urban land surface temperature[J]. Remote Sensing Technology and Application, 23(4): 405-409.]

陈小勇, 林鹏. 2000. 我国红树植物分布的空间自相关分析 [J]. 华东师范大学学报, (3): 104-109. [Chen X Y, Lin P. 2000. Spatial autocorrelation analysis on the distribution of mangrove in China[J]. Journal of East China Normal University, (3): 104-109.]

池腾龙, 曾坚, 王思粀. 2016. 基于 RS 和 GIS 的郑州市植被覆 盖度与地表温度演化研究 [J]. 中国园林, 32(10): 78-83. [Chi T L, Zeng J, Wang S T. 2016. Research of the relationship between vegetation fraction and evolution of thermal environment in Zhengzhou based on RS and GIS technology[J]. Chinese Landscape Architecture, 32(10): 78-83.]

韩贵锋, 梁保平. 2011. 地表温度与植被指数相关性的空间 尺度特征: 以重庆市为例 [J]. 中国园林, 27(1): 68-72.

[Han G F, Liang B P. 2011. Spatial scale characteristics of correlation between land surface tempe-rature and vegetation index: A case study of Chongqing City[J]. Chinese Landscape Architecture, 27(1): 68-72.]

李斌, 王慧敏, 秦明周, 等. 2017. NDVI、NDMI 与地表温度关 系的对比研究 [J]. 地理科学进展, 36(5): 589-596. [Li B, Wang H M, Qin M Z, et al. 2017. Comparative study on the correlations between NDVI, NDMI and LST[J]. Progress in Geography, 36(5): 585-596.]

李月臣, 杨华, 张锦水, 等. 2006. 土地利用/覆盖变化空间变 异特征与遥感影像空间分辨率的选取: 以北京地区为例 [J]. 干旱区地理, 29(4): 570-575. [Li Y C, Yang H, Zhang J S, et al. 2006. Spatial variation in landuse/cover change and choice of spatial resolution for remote sensing: A case study in Beijing[J]. Arid Land Geography, 29(4): 570575.]

马恭博. 2014. 莱州湾南岸土壤盐分时空变异性及运移模拟 研究 [D]. 青岛: 国家海洋局第一海洋研究所. [Ma G B. 2014. Soil salinity spatial- temporal variation and salinity transport simulation in southern Laizhou Bay[D]. Qingdao, China: The First Institute of Oceanography, State Oceanic Administration.]

倪万鬼, 牛富俊, 刘东燕. 2002. 黄土土性的空间自相关性研 究 $[J]$. 西安工程学院学报, 24(2): 4-9. [Ni W K, Niu F J, Liu D Y. 2002. A study on the spatial selfcorrelation of loess parameters[J]. Journal of Xi'an Engineering University, 24(2): 4-9.]

覃志豪, 李文娟, 徐斌, 等. 2004. 陆地卫星 TM6 波段范围内 地表比辐射率的估计 [J]. 国土资源遥感, (3): 28-32, 36, 41, 74. [Qin Z H, Li W J, Xu B, Chen Z X, Liu J. 2004. The estimation of land surface emissivity for Landsat TM6
[J]. Remote Sensing for Land \& Rescorces, (3): 28-32, 36, 41,74 .]

田平, 田光明, 王飞儿, 等. 2006. 基于 TM 影像的城市热岛效 应和植被覆盖指数关系研究 [J]. 科技通报, 22(5): 708713. [Tian P, Tian G M, Wang F E, et al. 2006. Urban heat island effect and vegetation cover index relation using Landsat TM image[J]. Bulletin of Science and Technology, 22(5): 708-713.]

王劲峰. 2006. 空间分析 [M]. 北京: 科学出版社, 76-84. [Wang J F. 2006. Spatial analysis[M]. Beijing, China: Science Press: 76-84.]

武鹏飞, 王茂军, 张学. 2009. 北京市植被绿度与城市热岛效 应关系研究 $[\mathrm{J}]$. 北京林业大学学报, 31(5): 54-60. [Wu P F, Wang M J, Zhang X. 2009. Relationship between vegetation greenness and urban heat island effect in Beijing[J]. Journal of Beijing Forestry University, 31(5): 54-60.]

冊亭, 张勇, 侯西勇. 2014. 基于半变异函数的环渤海地区 NDVI 空间格局特征研究 [J]. 生态科学, 33(2): 313-320. [Wu T, Zhang Y, Hou X Y. 2014. Analysis of spatial patterns of NDVI over the Circum-Bohai Sea region based on semi-variogram[J]. Ecological Science, 33(2): 313-320.]

谢苗苗, 王仰麟, 付梅臣. 2011. 城市地表温度热岛影响因素 研究进展 [J]. 地理科学进展, 30(1): 35-41. [Xie M M, Wang Y L, Fu M C. 2011. An overview and perspective about causative factors of surface urban heat island effects [J]. Progress in Geography, 30(1): 35-41.]

杨春华, 雷波, 张晟. 2013. 重庆市主城区热岛效应与植被覆 盖关系研究 [J]. 人民长江, 44(7): 51-55. [Yang C H, Lei B, Zhang S. 2013. Relationship between urban heat island effect and vegetation cover in core area of Chongqing City [J]. Yangtze River, 44(7): 51-55.]

游绚. 2011. 福州盆地城市热环境的时空格局演变及其尺度 效应研究 [D]. 福州: 福建师范大学. [You X. 2011. A study of spatiotemporal evolvement and scale effects on urban thermal environment in Fuzhou Basin[D]. Fuzhou, China: Fujian Normal University.]

游绚, 晏路明. 2009. 基于 ETM+影像辐射传导方程算法的地 表温度反演 [J]. 科技情报开发与经济, 19(27): 134-136, 138. [You X, Yan L M. 2009. Retrieving land surface temperature from the ETM+ image radioactive transfer equation $[\mathrm{J}]$. Sci-Tech Information Development and Economy, 19(27): 134-136, 138.]

张穗, 何报寅, 杜耘. 2003. 武汉市城区热岛效应的遥感研究 [J]. 长江流域资源与环境, 12(5): 445- 449. [Zhang S, He B Y, Du Y. 2003. Investigation on heat-island effection in Wuhan City zone by remote sensing $[\mathrm{J}]$. Resources and Environment in the Yangtze Basin, 12(5): 445- 449.]

张小飞, 王仰麟, 吴健生, 等. 2006. 城市地域地表温度-植被 
覆盖定量关系分析: 以深圳市为例 [J]. 地理研究, 25(3): 369-377, 561. [Zhang X F, Wang Y L, Wu J S, et al. 2006. Study on land surface temperature vegetation cover relationship in urban region: A case in Shenzhen City[J]. Geographical Research, 25(3): 369-377, 561.]

Bachmaier M, Backes M. 2011. Variogram or semivariogram? Variance or semivariance? Allan variance or introducing a new term[J]. Mathematical Geosciences, 43(6): 735-740.

Howard L. 1833. Climate of London deduced from meteorologieal observation[J]. Harvey and Darton, 1(3): 1-24.

Makoto Y, Robert D, Yoshitake K, et al. 2001. The cooling effect of paddy fields on summertime air temperature in residential Tokyo, Japan[J]. Landscape and Urban Planning, 53 (1): $17-27$.

Meisel, J E, Turner M G. 1998. Scale detection in real and artificial landscapes using semivariance analysis[J]. Landscape Ecology, 13(6): 347-362.

Mejia-dominguez N R, Meave J A, Díaz-Ávalos C. 2012. Spa- tial structure of the abiotic environment and its association with sapling community structure and dynamics in a cloud forest[J]. International Journal of Bio-meteorology, 56(2): 305-318.

Sokal R R, Oden N L. 1978. Spatial autocorrelation in biology: 1. Methodology[J]. Biological Journal of the Linnean Society, 10(2): 199-228.

Wartenberg D. 1985. Multivariate spatialcorrelation: A method for exploratory geographical analysis[J]. Geographical Analysis, 17(4): 263-283.

Weng Q H , Lu D, Schubring J. 2004. Estimation of land surface temperature-vegetation abundance relationship for urban heat island studies[J]. Remote Sensing of Environment, 89(4): 467-483.

Yuan F, Bauer M E. 2007. Comparision of impervious surface area and normalized difference vegetation index as indicators of surface urban heat island effects in landsat imagery [J]. Remote Sensing of Environment, 106(3): 375-386.

\title{
Scale effect of the spatial correlation between urban land surface temperature and NDVI
}

\author{
JIANG Yinghui ${ }^{1,2}$, JIAO Limin ${ }^{1,2 *}$, ZHANG Boen ${ }^{1,2}$ \\ (1. School of Resource and Environmental Sciences, Wuhan University, Wuhan 430079, China; 2. Key Laboratory \\ of Geographic Information System, Ministry of Education, Wuhan University, Wuhan 430079, China)
}

\begin{abstract}
The spatial correlation between urban land surface temperature (LST) and vegetation coverage (NDVI) has been widely studied, but its scale effect is often ignored, which brings uncertainty to the results. Taking Zhengzhou City as an example and based on four Landsat 8 images, this study retrieved the land surface temperature by the radiation conduction method, and identified the spatial correlation analysis scale of the land surface temperature by using the semivariance function. It then combined the spatial correlation index Moran's I to discuss the spatial correlation between land surface temperature and vegetation coverage from three aspects: multi-scales, multi-seasons, and multi-adjacent ranges. The results show that: (1) Both the univariate spatial autocorrelation scale and bivariate spatial correlation scale of LST and NDVI are around $300 \mathrm{~m}$; (2) Within the $300 \mathrm{~m}$ correlation scale, there is a significant scale effect in the univariate spatial autocorrelation, but the scale effect of bivariate spatial correlation is much weaker by comparison; (3) The univariate spatial autocorrelation and bivariate spatial correlation scale effects of LST and NDVI show significant seasonal differences; (4) With the increase of adjacent range, the spatial autocorrelation of LST and NDVI weakens, and the scale effect is more obvious. Therefore, to measure the spatial correlation between LST and NDVI, spatiotemporal scale effect should be taken into consideration. This study should be helpful for further understanding the scale effect of spatial correlation between LST and NDVI.
\end{abstract}

Key words: land surface temperature; NDVI; semi-variogram; spatial correlation; scale effect; Zhengzhou City 